\title{
Subgroup analyses of the safety and efficacy of ramucirumab in Japanese and Western patients in RAINBOW: a randomized clinical trial in second-line treatment of gastric cancer
}

\author{
Kohei Shitara $^{1} \cdot$ Kei Muro ${ }^{2}$ Y Yasuhiro Shimada ${ }^{3}$ Shuichi Hironaka ${ }^{4}$. \\ Naotoshi Sugimoto $^{5}$ - Yoshito Komatsu ${ }^{6}$ - Tomohiro Nishina ${ }^{7} \cdot$ Kensei Yamaguchi $^{8}$. \\ Yoshihiko Segawa $^{9}$ Yasushi Omuro ${ }^{10}$ - Takao Tamura ${ }^{11}$ - Toshihiko Doi ${ }^{1}$ • \\ Seigo Yukisawa ${ }^{12} \cdot$ Hirofumi Yasui $^{13} \cdot$ Fumio Nagashima $^{14} \cdot$ Masahiro Gotoh $^{15}$. $^{\circ}$ \\ Taito Esaki $^{16} \cdot$ Michael Emig $^{17} \cdot K_{\text {Kumari Chandrawansa }}{ }^{18} \cdot$ Astra M. Liepa $^{19}$. $^{2}$ \\ Hansjochen Wilke $^{20} \cdot$ Yukako Ichimiya $^{21} \cdot$ Atsushi Ohtsu $^{22}$
}

Received: 23 March 2015/Accepted: 8 October 2015/Published online: 28 October 2015

(c) The International Gastric Cancer Association and The Japanese Gastric Cancer Association 2015

\begin{abstract}
Background We evaluated the safety and efficacy of ramucirumab plus paclitaxel versus placebo plus paclitaxel in patients previously treated for advanced gastric or gastroesophageal junction adenocarcinoma in Japanese and Western subgroups from the RAINBOW trial.

Methods Patients received ramucirumab at $8 \mathrm{mg} / \mathrm{kg}$ or placebo (days 1 and 15) plus paclitaxel at $80 \mathrm{mg} / \mathrm{m}^{2}$ (days
\end{abstract}

This work was previously presented in part at the 50th Annual Meeting of the American Society of Clinical Oncology, Chicago, IL, May 30 to June 3, 2014; the 52nd Annual Meeting of the Japan Society of Clinical Oncology, Yokohama, Japan, August 28-30, 2014; and the 12th Annual Meeting of the Japanese Society of Medical Oncology, Fukuoka, Japan, July 17-19, 2014.

Kohei Shitara

kshitara@east.ncc.go.jp

1 Department of Gastroenterology and Gastrointestinal Oncology, National Cancer Center Hospital East, 6-5-1, Kashiwanoha, Kashiwa, Chiba 277-8577, Japan

2 Aichi Cancer Center, Nagoya, Japan

3 National Cancer Center Hospital, Tokyo, Japan

4 Chiba Cancer Center, Chiba, Japan

5 Osaka Medical Center for Cancer and Cardiovascular Diseases, Osaka, Japan

6 Hokkaido University Hospital, Sapporo, Japan

7 Shikoku Cancer Center, Matsuyama, Japan

8 Saitama Cancer Center, Kita-Adachi-Gun, Japan

9 Saitama Medical University International Medical Center, Hidaka, Japan

10 Tokyo Metropolitan Cancer and Infectious Diseases Center Komagome Hospital, Tokyo, Japan
1,8 , and 15 of a 28 -day cycle). End points were compared between treatment arms within Japanese $(N=140)$ and Western $(N=398)$ populations.

Results The incidence of adverse events of grade 3 or higher was higher for ramucirumab plus paclitaxel in both populations (Japanese population, $83.8 \%$ vs $52.1 \%$; Western population, $79.1 \%$ vs $61.9 \%$ ). Neutropenia was the commonest adverse event of grade 3 or higher, with a higher incidence for ramucirumab plus paclitaxel (Japanese population, $66.2 \%$ vs $25.4 \%$; Western population, $32.1 \%$ vs $14.7 \%$ ). The incidence of febrile neutropenia was low and was similar between treatment arms in both populations. The overall survival hazard ratio was $0.88(95 \%$

11 Kinki University Faculty of Medicine, Sayama, Japan

12 Tochigi Cancer Center, Utsunomiya, Japan

13 Shizuoka Cancer Center, Sunto-gun, Japan

14 Kyorin University Hospital, Tokyo, Japan

15 Osaka Medical College Hospital, Takatsuki, Japan

16 National Kyushu Cancer Center, Fukuoka, Japan

17 Eli Lilly and Company, Bad Homburg, Germany

18 Eli Lilly and Company, Bridgewater, NJ, USA

19 Eli Lilly and Company, Indianapolis, IN, USA

20 Kliniken Essen-Mitte Center of Palliative Care, Essen, Germany

21 Eli Lilly Japan K. K., Kobe, Japan

22 National Cancer Center, Tokyo, Japan 
confidence interval, 0.60-1.28) in the Japanese population and 0.73 (95\% confidence interval, 0.58-0.91) in the Western population. The progression-free survival hazard ratio was 0.50 (95\% confidence interval, $0.35-0.73$ ) in the Japanese population and 0.63 (95\% confidence interval, $0.51-0.79)$ in the Western population. The objective response rate was higher for ramucirumab plus paclitaxel in both populations (Japanese population, $41.2 \%$ vs $19.4 \%$; Western population, $26.8 \%$ vs $13.0 \%$ ), as was the 6-month survival rate (Japanese population, $94.1 \%$ vs $71.4 \%$; Western population, $66.0 \%$ vs $49.0 \%$ ).

Conclusions Safety profiles of the ramucirumab plus paclitaxel arm were similar between populations, though there was a higher incidence of neutropenia in Japanese patients. Progression-free survival and objective response rate improvements were observed for ramucirumab plus paclitaxel in both populations.

ClinicalTrials.gov identifier: NCT01170663

Keywords Advanced gastric or gastroesophageal junction adenocarcinoma - Japanese patients - Paclitaxel . Ramucirumab - Vascular endothelial growth factor receptor 2

\section{Introduction}

Gastric cancer is the fifth commonest malignancy and the third leading cause of cancer death worldwide [1]. In Japan, gastric cancer is the second commonest malignancy [1]. Currently, platinum-based and fluoropyrimidine-based combinations have been established worldwide as first-line regimens for treatment of advanced disease [2]. However, treatment options have been limited after failure of firstline therapy, resulting in short survival [3-5].

The RAINBOW trial was conducted (at 170 investigative sites in 27 countries) to compare the efficacy and safety of ramucirumab plus paclitaxel (RAM + PTX) with placebo plus paclitaxel (PL + PTX) in 665 randomly assigned (intent-to-treat) patients with metastatic or unresectable, locally advanced gastric or gastroesophageal junction adenocarcinoma whose disease had progressed during or following first-line fluoropyrimidine- and platinum-containing chemotherapy. In this study, overall survival (OS), progression-free survival (PFS), and the objective response rate (ORR) were all improved with statistical significance in patients allocated to receive RAM + PTX compared with patients allocated to receive PL + PTX [6].

Recent randomized clinical trials exploring second-line or later single-agent chemotherapy versus best supportive care or comparing two single-agent chemotherapies in gastric cancer have suggested better survival outcome in
Japanese patients in comparison with Western patients [3, $4,7]$, although this is not a universal phenomenon in all Asian patients [5]. Differences in survival outcome between Japanese and Western patients with gastric cancer are well known, and the reasons have been widely speculated upon and include tumor biology, ethnicity, health care, insurance or reimbursement systems, and use of subsequent therapy $[8,9]$. In this article, we compare the outcomes of Japanese versus Western patients in the RAINBOW trial.

\section{Patients and methods}

\section{Study design and patients}

The study design and procedures for RAINBOW have been previously published [6]. Eligible patients had metastatic or unresectable, locally advanced gastric or gastroesophageal junction adenocarcinoma and documented objective radiological or clinical disease progression during or within 4 months of the last dose of first-line platinum-based and fluoropyrimidine-based doublet therapy with or without anthracycline, and an Eastern Cooperative Oncology Group (ECOG) performance status (PS) of 0 or 1.

Each center's institutional review board or independent ethics committee approved the study. The trial followed the guiding principles of the Declaration of Helsinki and the good clinical practice guidelines of the International Conference on Harmonisation of Technical Requirements for Registration of Pharmaceuticals for Human Use. All patients provided written informed consent.

\section{Treatment}

Patients were randomly assigned, in a 1:1 ratio, to receive either ramucirumab at $8 \mathrm{mg} / \mathrm{kg}$ or placebo intravenously on days 1 and 15 plus paclitaxel at $80 \mathrm{mg} / \mathrm{m}^{2}$ intravenously on days 1,8 , and 15 of a 28 -day cycle [6]. Randomization was stratified by three factors: geographic region (region 1, Australia, Europe, Israel, and USA; region 2, Argentina, Brazil, Chile, and Mexico; region 3, Hong Kong, Japan, Singapore, South Korea, and Taiwan); time to progression (TTP) from the start of first-line chemotherapy (less than 6 months vs 6 months or more); and disease measurability (measurable vs nonmeasurable disease).

\section{Statistical analysis}

In this subgroup analysis, we compared treatment arms (RAM + PTX vs PL + PTX) within Japanese and Western (Australia, Europe, Israel, and the USA) populations. 
The comparison between Japanese and Western populations was not part of the statistical analysis plan and was done on a post hoc basis.

For OS and PFS, survival curves were generated by the Kaplan-Meier method. The hazard ratio (HR) was estimated with the $95 \%$ confidence interval (CI) by means of a Cox proportional hazards model stratified by TTP from the start of first-line chemotherapy and disease measurability. Post hoc exploratory analyses were also conducted to evaluate the impact of post-discontinuation systemic therapy (PDT) on OS, evaluating the patients with PDT and without PDT separately. ORR and disease control rate (DCR) were estimated with $95 \%$ CIs. DCR was defined as the proportion of patients with complete response, partial response, or stable disease. For the patients with measurable disease, the percent change in tumor size from the baseline to the smallest postbaseline value is presented as a waterfall plot. Disease progression and tumor response were assessed by investigators in accordance with the Response Evaluation Criteria In Solid Tumors version 1.1. For every postbaseline assessment, European Organization for Research and Treatment of Cancer quality-of-life questionnaire (EORTC QLQ-C30, version 3.0) [10] scores were classified as improved or worsened if the patient's change from the baseline was 10 points or more (on a 100-point scale); a change of less than 10 points was classified as stable. The time to deterioration in ECOG PS was estimated from randomization to the first worsening to an ECOG PS of 2 or higher, and was analyzed with a Cox model. Efficacy, quality-of-life, and PS analyses included intent-to-treat patients in the Japanese and Western populations.

Safety analyses included patients who received at least one dose of any drug. Adverse events were graded in accordance with the National Cancer Institute Common Terminology Criteria for Adverse Events (version 4.02).

This trial is registered with ClinicalTrials.gov (identifier NCT01170663).

\section{Results}

\section{Patients}

The Japanese population consisted of 140 randomly assigned patients (RAM + PTX arm, 68 patients; PL + PTX arm, 72 patients). The Western population consisted of 398 randomly assigned patients from Australia, Europe, Israel, and the USA (RAM + PTX arm, 198 patients; PL + PTX arm, 200 patients).

Figure 1 shows the trial profile in the Japanese and Western populations from RAINBOW. Baseline patient and tumor characteristics were generally balanced between the treatment arms within each population. Several characteristics were unequally distributed between the Japanese and Western populations (Table 1). The percentage of patients with the following characteristics was higher in the Japanese population: ECOG PS 0, TTP from the start of first-line therapy of 6 months or more, progression during first-line therapy, gastric cancer (vs gastroesophageal junction cancer), zero to two metastatic sites (vs three or more), presence of ascites, diffuse-type adenocarcinoma, and prior doublet treatment.

The median duration of study therapy in the RAM + PTX arm was longer than in the PL + PTX arm in both populations (Japanese population, 22.5 weeks vs 12.0 weeks; Western population, 16.1 weeks vs 12.0 weeks); the longer duration of treatment relates to the longer PFS in the combination therapy arm. The median relative dose intensity of ramucirumab was 97.6 and $98.6 \%$ in the Japanese and Western populations, respectively.

The median relative dose intensity of paclitaxel in the RAM + PTX arm was lower than that in the PL + PTX arm in the Japanese population (73.6\% vs $91.4 \%$ ), and was similar in the Western population (89.7\% vs $93.5 \%)$. However, the median cumulative dose of paclitaxel in the RAM + PTX arm was higher than in the PL + PTX arm in the Japanese population $\left(1026 \mathrm{mg} / \mathrm{m}^{2}\right.$ vs $\left.715 \mathrm{mg} / \mathrm{m}^{2}\right)$ and the Western population $\left(802 \mathrm{mg} / \mathrm{m}^{2}\right.$ vs $\left.599 \mathrm{mg} / \mathrm{m}^{2}\right)$.

\section{Safety}

The safety population in this subgroup analysis comprised 139 patients from Japan (RAM + PTX arm, 68 patients; PL + PTX arm, 71 patients) and 393 patients from the West (RAM + PTX arm, 196 patients; PL + PTX arm, 197 patients). The commonest treatment-emergent adverse events (TEAEs) of any grade occurring with a higher incidence in the RAM + PTX arm compared with the PL + PTX arm in both populations included fatigue, neutropenia, neuropathy, decreased appetite, and epistaxis (Table 2). The incidence of TEAEs of grade 3 or higher was higher in the RAM + PTX arm than in PL + PTX arm in both populations (Japanese population, $83.8 \%$ vs $52.1 \%$; Western population, $79.1 \%$ vs $61.9 \%$ ).

Neutropenia was the commonest TEAE of grade 3 or higher occurring with a higher incidence in the RAM + PTX arm than in the PL + PTX arm in both populations (Japanese population, $66.2 \%$ vs $25.4 \%$; Western population, $32.1 \%$ vs $14.7 \%$ ). The incidence of febrile neutropenia was low and similar between treatment arms (RAM + PTX vs PL + PTX) in both populations (Japanese population, $4.4 \%$ vs $4.2 \%$; Western population, $2.6 \%$ vs $1.5 \%)$. 


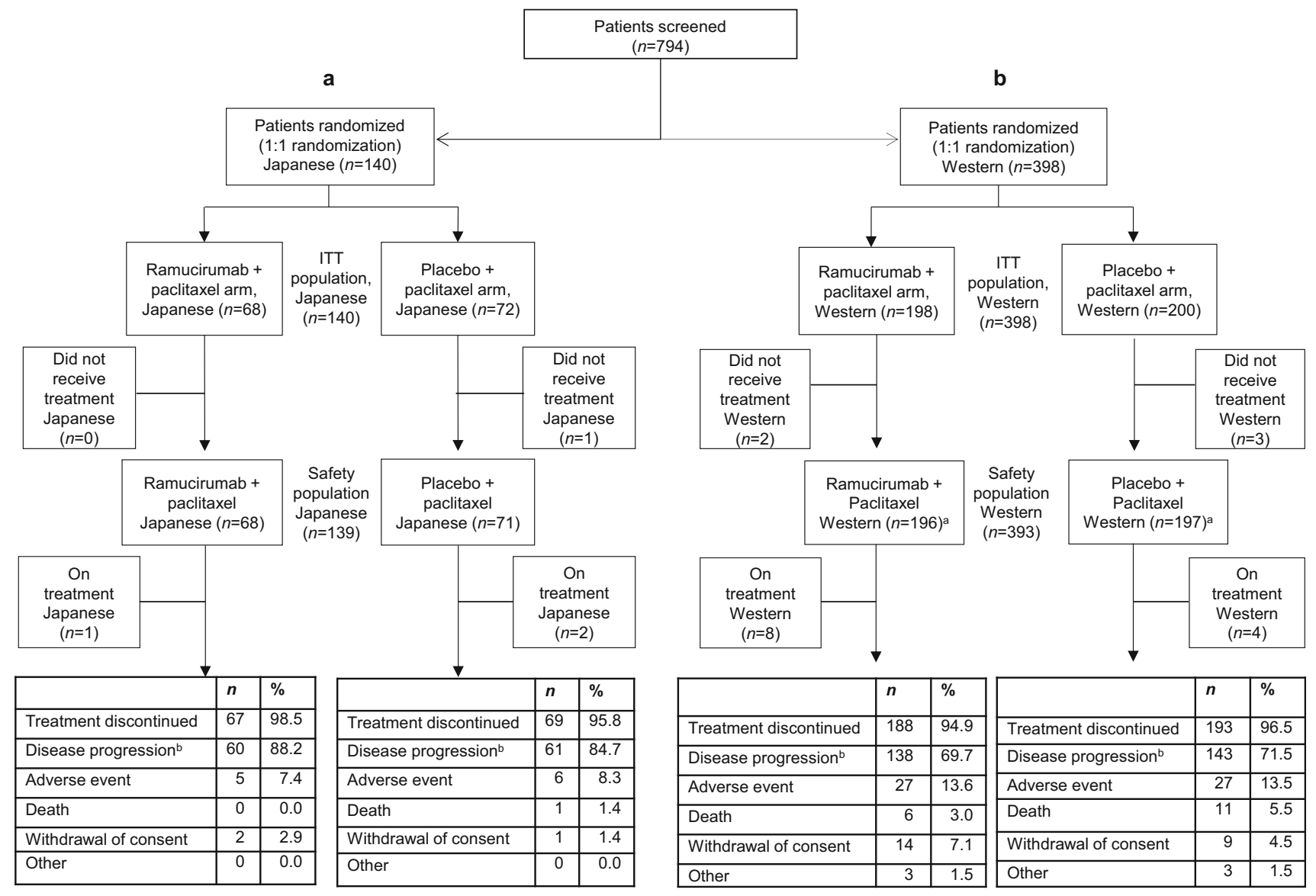

Fig. 1 Trial profile for the RAINBOW Japanese population (a) and Western population (b). ITT intent to treat, superscript $a$ one patient was randomized to the placebo group but received only one dose of

ramucirumab, superscript $b$ progressive disease-either radiographic progression or symptomatic deterioration

With the exception of hypertension, all adverse events of special interest of grade 3 or higher were reported with an incidence of less than $5 \%$ across treatment arms and populations (Table 2). The incidences of grade 3 or higher bleeding/hemorrhage (Japanese population, $4.4 \%$ vs $1.4 \%$; Western population, $4.6 \%$ vs $1.5 \%$ ) and gastrointestinal perforation (Japanese population, $1.5 \%$ vs $0.0 \%$; Western population, $1.0 \%$ vs $0.0 \%$ ) were higher in the RAM + PTX arm than in the PL + PTX arm and were similar in both populations. Grade 3 proteinuria (RAM + PTX vs PL + PTX) was reported only in the Japanese population ( $4.4 \%$ vs $0.0 \%$ ). Grade 3 hypertension was more frequently reported in the Western population (Japanese population, $4.4 \%$ vs $0.0 \%$; Western population, $18.9 \%$ vs $2.5 \%$ ). No grade 3 or higher proteinuria or hypertension was reported in the Japanese population or the Western population.

The percentage of patients in the Japanese population who experienced at least one serious adverse event was similar between the treatment arms (RAM + PTX vs PL + PTX) and lower than in the Western population

[Japanese population, $22.1 \%$ (15) vs $26.8 \%$ (19); Western population, $53.6 \%$ (105) vs $43.7 \%$ (86)]. The incidence of TEAEs leading to death was similar between the treatment arms for both the Japanese population [1.5 \% (1) vs $2.8 \%$ (2)] and the Western population [12.8\% (25) vs $19.3 \%$ (38)].

\section{Treatment discontinuation}

Disease progression was the commonest reason for treatment discontinuation in both treatment populations (Fig. 1): 88.2 and $84.7 \%$ of patients in the Japanese population (RAM + PTX arm and PL + PTX arm, respectively), and 69.7 and $71.5 \%$ of patients in the Western population (RAM + PTX arm and PL + PTX arm, respectively). The second commonest reason for treatment discontinuation was adverse events, occurring in 7.4 and $8.3 \%$ of patients in the Japanese population (RAM + PTX arm and PL + PTX arm, respectively) and in 13.6 and $13.5 \%$ of patients in the Western population (RAM + PTX arm and PL + PTX arm, respectively). 
Table 1 Patient and tumor characteristics of the intent-to-treat population at the baseline

\begin{tabular}{|c|c|c|c|c|c|c|}
\hline & \multicolumn{3}{|c|}{ Japanese patients } & \multicolumn{3}{|c|}{ Western patients } \\
\hline & $\begin{array}{l}\text { RAM + PTX } \\
(n=68)\end{array}$ & $\begin{array}{l}\mathrm{PL}+\mathrm{PTX} \\
(n=72)\end{array}$ & $\begin{array}{l}\text { Total } \\
(N=140)\end{array}$ & $\begin{array}{l}\text { RAM + PTX } \\
(n=198)\end{array}$ & $\begin{array}{l}\mathrm{PL}+\mathrm{PTX} \\
(n=200)\end{array}$ & $\begin{array}{l}\text { Total } \\
(N=398)\end{array}$ \\
\hline Male sex & $46(67.6 \%)$ & $53(73.6 \%)$ & $99(70.7 \%)$ & $140(70.7 \%)$ & $147(73.5 \%)$ & $287(72.1 \%)$ \\
\hline \multicolumn{7}{|l|}{ Age } \\
\hline Median (years) & 64.0 & 64.5 & 64.0 & 60.0 & 61.0 & 60.0 \\
\hline Range (years) & $34-76$ & $29-76$ & $29-76$ & $25-83$ & $24-84$ & $24-84$ \\
\hline$<65$ years & $36(52.9 \%)$ & $36(50.0 \%)$ & $72(51.4 \%)$ & $123(62.1 \%)$ & $126(63.0 \%)$ & $249(62.6 \%)$ \\
\hline$\geq 65$ years & $32(47.1 \%)$ & $36(50.0 \%)$ & $68(48.6 \%)$ & $75(37.9 \%)$ & $74(37.0 \%)$ & $149(37.4 \%)$ \\
\hline \multicolumn{7}{|l|}{ ECOG performance status } \\
\hline 0 & $37(54.4 \%)$ & $43(59.7 \%)$ & $80(57.1 \%)$ & $68(34.3 \%)$ & $75(37.5 \%)$ & $143(35.9 \%)$ \\
\hline 1 & $31(45.6 \%)$ & $29(40.3 \%)$ & $60(42.9 \%)$ & $130(65.7 \%)$ & $125(62.5 \%)$ & $255(64.1 \%)$ \\
\hline \multicolumn{7}{|l|}{ Weight loss (prior 3 months) } \\
\hline$<10 \%$ & $61(89.7 \%)$ & $63(87.5 \%)$ & $124(88.6 \%)$ & $168(84.8 \%)$ & $169(84.5 \%)$ & $337(84.7 \%)$ \\
\hline$\geq 10 \%$ & $7(10.3 \%)$ & $9(12.5 \%)$ & $16(11.4 \%)$ & $30(15.2 \%)$ & $29(14.5 \%)$ & $59(14.8 \%)$ \\
\hline $\begin{array}{l}\text { Time to PD with } 1 \text { st-line therapy }{ }^{\mathrm{a}} \geq 6 \\
\text { months }\end{array}$ & $37(54.4 \%)$ & $37(51.4 \%)$ & $74(52.9 \%)$ & $72(36.4 \%)$ & $75(37.5 \%)$ & $147(36.9 \%)$ \\
\hline Progression during 1st-line therapy & $63(92.6 \%)$ & $63(87.5 \%)$ & $126(90.0 \%)$ & $119(60.1 \%)$ & $112(56.0 \%)$ & $231(58.0 \%)$ \\
\hline \multicolumn{7}{|l|}{ Primary tumor } \\
\hline Gastric & $65(95.6 \%)$ & $65(90.3 \%)$ & $130(92.9 \%)$ & $139(70.2 \%)$ & $137(68.5 \%)$ & $276(69.3 \%)$ \\
\hline GEJ & $3(4.4 \%)$ & $7(9.7 \%)$ & $10(7.1 \%)$ & $59(29.8 \%)$ & $63(31.5 \%)$ & $122(30.7 \%)$ \\
\hline Present at study entry & $44(64.7 \%)$ & $52(72.2 \%)$ & $96(68.6 \%)$ & $130(65.7 \%)$ & $124(62.0 \%)$ & $254(63.8 \%)$ \\
\hline Measurable disease & $49(72.1 \%)$ & $52(72.2 \%)$ & $101(72.1 \%)$ & $164(82.8 \%)$ & $168(84.0 \%)$ & $332(83.4 \%)$ \\
\hline \multicolumn{7}{|l|}{ Histologic subtype } \\
\hline Intestinal & $29(42.6 \%)$ & $24(33.3 \%)$ & $53(37.9 \%)$ & $92(46.5 \%)$ & $86(43.0 \%)$ & $178(44.7 \%)$ \\
\hline Diffuse & $32(47.1 \%)$ & $43(59.7 \%)$ & $75(53.6 \%)$ & $62(31.3 \%)$ & $72(36.0 \%)$ & $134(33.7 \%)$ \\
\hline \multicolumn{7}{|l|}{ Metastases } \\
\hline $0-2$ sites & $54(79.4 \%)$ & $54(75.0 \%)$ & $108(77.1 \%)$ & $110(55.6 \%)$ & $129(64.5 \%)$ & $239(60.1 \%)$ \\
\hline$\geq 3$ sites & $14(20.6 \%)$ & $18(25.0 \%)$ & $32(22.9 \%)$ & $88(44.4 \%)$ & $71(35.5 \%)$ & $159(39.9 \%)$ \\
\hline Peritoneal metastasis & $36(52.9 \%)$ & $35(48.6 \%)$ & $71(50.7 \%)$ & $89(44.9 \%)$ & $87(43.5 \%)$ & $176(44.2 \%)$ \\
\hline Presence of ascites & $33(48.5 \%)$ & $25(34.7 \%)$ & $58(41.4 \%)$ & $67(33.8 \%)$ & $57(28.5 \%)$ & $124(31.2 \%)$ \\
\hline \multicolumn{7}{|l|}{ Prior cytotoxic therapy } \\
\hline $\begin{array}{l}\text { Triplet therapy: platinum/ } \\
\text { fluoropyrimidine with anthracycline }\end{array}$ & $0(0 \%)$ & $0(0 \%)$ & $0(0 \%)$ & $68(34.3 \%)$ & $80(40.0 \%)$ & $148(37.2 \%)$ \\
\hline $\begin{array}{l}\text { Doublet therapy: platinum/ } \\
\text { fluoropyrimidine }\end{array}$ & $68(100.0 \%)$ & $72(100.0 \%)$ & $140(100.0 \%)$ & $129(65.2 \%)$ & $118(59.0 \%)$ & $247(62.1 \%)$ \\
\hline $\begin{array}{l}\text { Prior targeted therapy (HER-2, EGFR, } \\
\text { other) }\end{array}$ & $8(11.8 \%)$ & $8(11.1 \%)$ & $16(11.4 \%)$ & $20(10.1 \%)$ & $15(7.5 \%)$ & $35(8.8 \%)$ \\
\hline Previous surgery for gastric cancer & $29(42.6 \%)$ & $20(27.8 \%)$ & $49(35.0 \%)$ & $70(35.4 \%)$ & $77(38.5 \%)$ & $147(36.9 \%)$ \\
\hline Total gastrectomy & $14(20.6 \%)$ & $8(11.1 \%)$ & $22(15.7 \%)$ & $28(14.1 \%)$ & $41(20.5 \%)$ & $69(17.3 \%)$ \\
\hline Partial gastrectomy & $15(22.1 \%)$ & $12(16.7 \%)$ & $27(19.3 \%)$ & $41(20.7 \%)$ & $34(17.0 \%)$ & $75(18.8 \%)$ \\
\hline Other & $0(0 \%)$ & $0(0 \%)$ & $0(0 \%)$ & $1(0.5 \%)$ & $2(1.0 \%)$ & $3(0.8 \%)$ \\
\hline
\end{tabular}

ECOG Eastern Cooperative Oncology Group, EGFR epidermal growth factor receptor, GEJ gastroesophageal junction, $H E R$-2 human epidermal growth factor receptor 2, $P D$ progressive disease, $P L$ placebo, $P T X$ paclitaxel, $R A M$ ramucirumab

a Time to PD is defined as the time from the start of first-line therapy until PD

\section{Post-discontinuation therapy}

Whereas the rate of PDT was balanced between the two treatments arms in both populations, the rate of PDT was higher in the Japanese population $(75.0 \%)$ than in the Western population (37.2\%). Additionally, a greater proportion of patients in the Japanese population $(25.0 \%$ in the RAM + PTX arm and $34.7 \%$ in the PL + PTX arm) 
Table 2 Treatment-emergent adverse events (TEAEs)

\begin{tabular}{|c|c|c|c|c|c|c|c|c|}
\hline \multirow[t]{3}{*}{ Preferred term ${ }^{\mathrm{a}}$} & \multicolumn{4}{|c|}{ Japanese patients } & \multicolumn{4}{|c|}{ Western patients } \\
\hline & \multicolumn{2}{|c|}{$\mathrm{RAM}+\mathrm{PTX}(n=68)$} & \multicolumn{2}{|c|}{$\mathrm{PL}+\operatorname{PTX}(n=71)$} & \multicolumn{2}{|c|}{$\mathrm{RAM}+\operatorname{PTX}(n=196)$} & \multicolumn{2}{|c|}{$\mathrm{PL}+\operatorname{PTX}(n=197)$} \\
\hline & Any grade & Grade $\geq 3$ & Any grade & Grade $\geq 3$ & Any grade & Grade $\geq 3$ & Any grade & Grade $\geq 3$ \\
\hline \multicolumn{9}{|c|}{ TEAEs (and febrile neutropenia) occurring in $20 \%$ or more of patients in the RAM + PTX arm } \\
\hline Any TEAE & $68(100.0 \%)$ & $57(83.8 \%)$ & $70(98.6 \%)$ & $37(52.1 \%)$ & $193(98.5 \%)$ & $155(79.1 \%)$ & $191(97.0 \%)$ & $\begin{array}{l}122 \\
\quad(61.9 \%)\end{array}$ \\
\hline Neutropenia $^{\mathrm{a}}$ & $58(85.3 \%)$ & $45(66.2 \%)$ & $37(52.1 \%)$ & $18(25.4 \%)$ & $84(42.9 \%)$ & $63(32.1 \%)$ & $48(24.4 \%)$ & $\begin{array}{l}29 \\
(14.7 \%)\end{array}$ \\
\hline Febrile neutropenia & $3(4.4 \%)$ & $3(4.4 \%)$ & $3(4.2 \%)$ & $3(4.2 \%)$ & $5(2.6 \%)$ & $5(2.6 \%)$ & $3(1.5 \%)$ & $3(1.5 \%)$ \\
\hline Leukopenia $^{\mathrm{a}}$ & $49(72.1 \%)$ & $31(45.6 \%)$ & $33(46.5 \%)$ & $10(14.1 \%)$ & $43(21.9 \%)$ & $19(9.7 \%)$ & $21(10.7 \%)$ & $8(4.1 \%)$ \\
\hline Neuropathy ${ }^{a}$ & $48(70.6 \%)$ & $3(4.4 \%)$ & $39(54.9 \%)$ & $4(5.6 \%)$ & $75(38.3 \%)$ & $22(11.2 \%)$ & $59(29.9 \%)$ & $11(5.6 \%)$ \\
\hline Decreased appetite & $37(54.4 \%)$ & $2(2.9 \%)$ & $33(46.5 \%)$ & $4(5.6 \%)$ & $70(35.7 \%)$ & $5(2.6 \%)$ & $54(27.4 \%)$ & $5(2.5 \%)$ \\
\hline Epistaxis & $37(54.4 \%)$ & $0(0 \%)$ & $10(14.1 \%)$ & $0(0 \%)$ & $53(27.0 \%)$ & $0(0 \%)$ & $13(6.6 \%)$ & $0(0 \%)$ \\
\hline Fatigue $^{\mathrm{a}}$ & $33(48.5 \%)$ & $1(1.5 \%)$ & $28(39.4 \%)$ & $2(2.8 \%)$ & $128(65.3 \%)$ & $33(16.8 \%)$ & $95(48.2 \%)$ & $13(6.6 \%)$ \\
\hline Diarrhea & $29(42.6 \%)$ & $3(4.4 \%)$ & $24(33.8 \%)$ & $2(2.8 \%)$ & $55(28.1 \%)$ & $5(2.6 \%)$ & $37(18.8 \%)$ & $2(1.0 \%)$ \\
\hline Vomiting & $21(30.9 \%)$ & $1(1.5 \%)$ & $14(19.7 \%)$ & $1(1.4 \%)$ & $50(25.5 \%)$ & $5(2.6 \%)$ & $39(19.8 \%)$ & $8(4.1 \%)$ \\
\hline Proteinuria & $20(29.4 \%)$ & $3(4.4 \%)$ & $5(7.0 \%)$ & $0(0 \%)$ & $22(11.2 \%)$ & $0(0 \%)$ & $11(5.6 \%)$ & $0(0 \%)$ \\
\hline Peripheral edema & $19(27.9 \%)$ & $0(0 \%)$ & $10(14.1 \%)$ & $1(1.4 \%)$ & $50(25.5 \%)$ & $3(1.5 \%)$ & $28(14.2 \%)$ & $1(0.5 \%)$ \\
\hline Hypertension $^{\mathrm{a}}$ & $16(23.5 \%)$ & $3(4.4 \%)$ & $1(1.4 \%)$ & $0(0 \%)$ & $52(26.5 \%)$ & $35(17.9 \%)$ & $11(5.6 \%)$ & $4(2.0 \%)$ \\
\hline Abdominal pain ${ }^{\mathrm{a}}$ & $7(10.3 \%)$ & $0(0 \%)$ & $10(14.1 \%)$ & $0(0 \%)$ & $81(41.3 \%)$ & $14(7.1 \%)$ & $61(31.0 \%)$ & $9(4.6 \%)$ \\
\hline \multicolumn{9}{|c|}{ Adverse events of special interest ${ }^{b}$} \\
\hline $\begin{array}{l}\text { Patients with any } \\
\text { adverse events of } \\
\text { special interest }\end{array}$ & $53(77.9 \%)$ & $11(16.2 \%)$ & $26(36.6 \%)$ & $3(4.2 \%)$ & $121(61.7 \%)$ & $55(28.1 \%)$ & $68(34.5 \%)$ & $19(9.6 \%)$ \\
\hline $\begin{array}{l}\text { Bleeding/hemorrhage } \\
\text { events }\end{array}$ & $46(67.6 \%)$ & $3(4.4 \%)$ & $19(26.8 \%)$ & $1(1.4 \%)$ & $72(36.7 \%)$ & $9(4.6 \%)$ & $28(14.2 \%)$ & $3(1.5 \%)$ \\
\hline Proteinuria & $20(29.4 \%)$ & $3(4.4 \%)$ & $5(7.0 \%)$ & $0(0 \%)$ & $23(11.7 \%)$ & $0(0 \%)$ & $11(5.6 \%)$ & $0(0 \%)$ \\
\hline Liver injury/failure & $16(23.5 \%)$ & $2(2.9 \%)$ & $10(14.1 \%)$ & $1(1.4 \%)$ & $22(11.2 \%)$ & $9(4.6 \%)$ & $20(10.2 \%)$ & $6(3.0 \%)$ \\
\hline Hypertension & $16(23.5 \%)$ & $3(4.4 \%)$ & $1(1.4 \%)$ & $0(0 \%)$ & $56(28.6 \%)$ & $37(18.9 \%)$ & $14(7.1 \%)$ & $5(2.5 \%)$ \\
\hline $\begin{array}{l}\text { Gastrointestinal } \\
\text { hemorrhage events }\end{array}$ & $8(11.8 \%)$ & $2(2.9 \%)$ & $4(5.6 \%)$ & $1(1.4 \%)$ & $20(10.2 \%)$ & $9(4.6 \%)$ & $12(6.1 \%)$ & $3(1.5 \%)$ \\
\hline $\begin{array}{l}\text { Infusion-related } \\
\text { reaction }\end{array}$ & $2(2.9 \%)$ & $0(0 \%)$ & $3(4.2 \%)$ & $0(0 \%)$ & $14(7.1 \%)$ & $2(1.0 \%)$ & $7(3.6 \%)$ & $0(0 \%)$ \\
\hline Renal failure & $4(5.9 \%)$ & $0(0 \%)$ & $4(5.6 \%)$ & $0(0 \%)$ & $12(6.1 \%)$ & $3(1.5 \%)$ & $7(3.6 \%)$ & $2(1.0 \%)$ \\
\hline $\begin{array}{l}\text { Congenital heart } \\
\text { failure }\end{array}$ & $3(4.4 \%)$ & $1(1.5)$ & $0(0 \%)$ & $0(0 \%)$ & $3(1.5 \%)$ & 0 & $2(1.0 \%)$ & $2(1.0 \%)$ \\
\hline $\begin{array}{l}\text { Venous } \\
\text { thromboembolic } \\
\text { events }\end{array}$ & $4(5.9 \%)$ & $2(2.9 \%)$ & $3(4.2 \%)$ & $2(2.8 \%)$ & $9(4.6 \%)$ & $6(3.1 \%)$ & $11(5.6 \%)$ & $6(3.0 \%)$ \\
\hline $\begin{array}{l}\text { Arterial } \\
\text { thromboembolic } \\
\text { events }\end{array}$ & $1(1.5 \%)$ & $0(0 \%)$ & $0(0 \%)$ & $0(0 \%)$ & $4(2.0 \%)$ & $2(1.0 \%)$ & $2(1.0 \%)$ & $1(0.5 \%)$ \\
\hline $\begin{array}{l}\text { Gastrointestinal } \\
\text { perforation }\end{array}$ & $1(1.5 \%)$ & $1(1.5 \%)$ & $0(0 \%)$ & $0(0 \%)$ & $2(1.0 \%)$ & $2(1.0 \%)$ & $1(0.5 \%)$ & $0(0 \%)$ \\
\hline
\end{tabular}

$P L$ placebo, $P T X$ paclitaxel, $R A M$ ramucirumab

${ }^{a}$ Consolidated TEAE terms comprise synonymous Medical Dictionary for Regulatory Activities preferred terms: fatigue includes asthenia; neutropenia includes decreased neutrophil count; neuropathy includes peripheral sensory neuropathy, paraesthesia, peripheral neuropathy, polyneuropathy, hypoasethesia, neuralgia, and dysesthesia; abdominal pain includes upper abdominal pain and lower abdominal pain; leukopenia includes decreased white blood cell; hypertension includes increased blood pressure, hypertensive cardiomyopathy, procedural hypertension, and systolic hypertension

${ }^{\mathrm{b}}$ Pooled adverse-event terms. Events pooled as gastrointestinal hemorrhage are also pooled as bleeding/hemorrhage. 
received fourth-line therapy or subsequent lines of therapy compared with in the Western population $(13.6 \%$ in the RAM + PTX arm and $7.0 \%$ in the PL + PTX arm). The most commonly used chemotherapy agents in both populations included irinotecan, taxanes, fluoropyrimidines, and platinum compounds.

\section{Efficacy}

OS, PFS, and ORR for the Japanese and Western populations are summarized in Fig. 2 and Table 3.

In the Japanese population, the HR for OS was 0.88 (95\% CI, 0.60-1.28). The median survival was 11.4 months [interquartile range (IQR), 7.9-19.4 months] in the RAM + PTX arm versus 11.5 months (IQR, 4.8-18.9 months) in the PL + PTX arm (Fig. 2a). The 6-month survival rate was $94.1 \%$ in

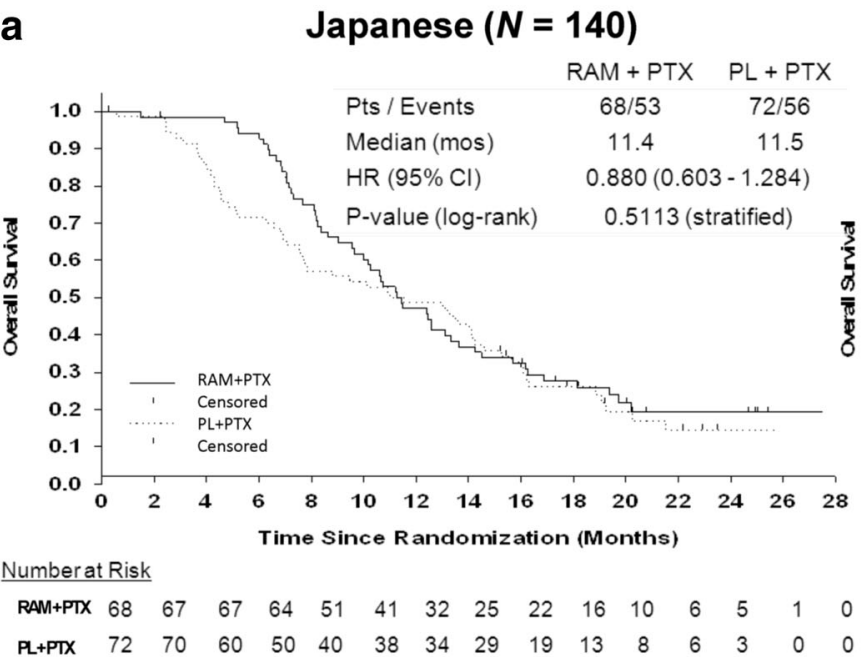

the RAM + PTX arm versus $71.4 \%$ in the PL + PTX arm in the Japanese population. In the Western population, the HR for OS was 0.73 (95\% CI, 0.58-0.91). The median survival was 8.6 months (IQR, 4.7-13.6 months) in the RAM + PTX arm versus 5.9 months (IQR, 3.1-11.0 months) in the PL + PTX arm (Fig. 2b). The 6-month survival rate was $66.0 \%$ in the RAM + PTX arm versus $49.0 \%$ in the PL + PTX arm in the Western population.

The OS by PDT use is shown in Fig. 3. Independent of the treatment arm, patients who did not receive PDT had shorter survival than patients who received any PDTs. In patients who did not receive PDT, there was a clear difference in OS between the two treatment arms (Fig. 3a, b), whereas in patients who received any PDT, the difference in survival was smaller (Fig. 3c, d) in both the Japanese population and the Western population.

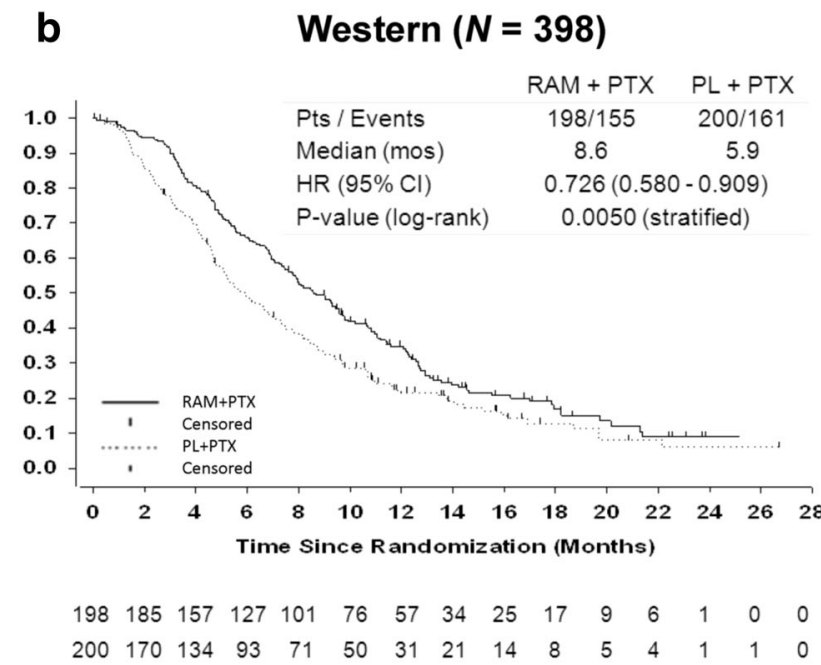

C

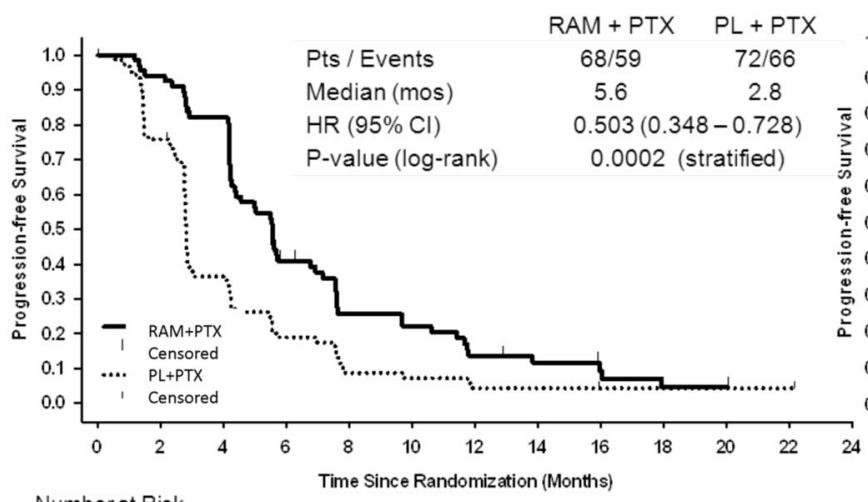

Number at Risk

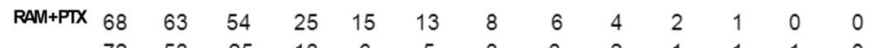

d

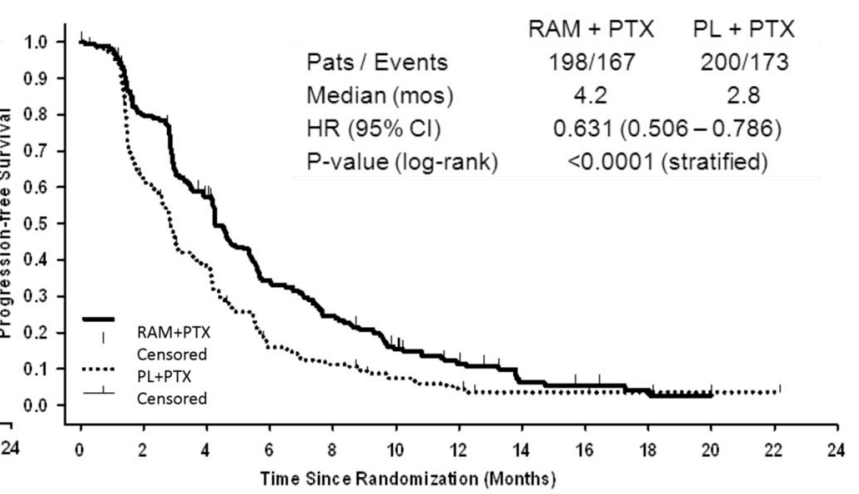

Fig. 2 Kaplan-Meier estimates of overall survival for Japanese patients (a) and Western patients (b) and of progression-free survival for Japanese patients (c) and Western patients (d). The analyses presented here are exploratory in nature. $C I$ confidence interval, $H R$ hazard ratio, mos months, $P L$ placebo, Pts patients, $P T X$ paclitaxel, $R A M$ ramucirumab 
Table 3 Objective tumor response

\begin{tabular}{|c|c|c|c|c|c|c|}
\hline & \multicolumn{3}{|l|}{ Japanese } & \multicolumn{3}{|l|}{ Western } \\
\hline & $\begin{array}{l}\text { RAM + PTX } \\
(n=68)\end{array}$ & $\begin{array}{l}\mathrm{PL}+\mathrm{PTX} \\
(n=72)\end{array}$ & $P$ value* & $\begin{array}{l}\mathrm{RAM}+\mathrm{PTX} \\
(n=198)\end{array}$ & $\begin{array}{l}\mathrm{PL}+\mathrm{PTX} \\
(n=200)\end{array}$ & $P$ value* \\
\hline \multicolumn{7}{|l|}{ Best overall response, $n(\%)$} \\
\hline Complete response (CR) & $1(1.5)$ & $0(0)$ & & $1(0.5)$ & $1(0.5)$ & \\
\hline Partial response (PR) & $27(39.7)$ & $14(19.4)$ & & $52(26.3)$ & $25(12.5)$ & \\
\hline Stable disease (SD) & $36(52.9)$ & $40(55.6)$ & & $99(50.0)$ & $87(43.5)$ & \\
\hline Progressive disease (PD) & $4(5.9)$ & $16(22.2)$ & & $32(16.2)$ & $57(28.5)$ & \\
\hline $\begin{array}{l}\text { Not evaluable/No tumor response } \\
\text { evaluation }\end{array}$ & $0(0)$ & $2(2.8)$ & & $14(7.1)$ & $30(15.0)$ & \\
\hline $\begin{array}{l}\text { Objective response rate: } \mathrm{CR}+\mathrm{PR}, \\
n(\%)\end{array}$ & $28(41.2)$ & $14(19.4)$ & 0.0035 & $53(26.8)$ & $26(13.0)$ & 0.0004 \\
\hline $95 \% \mathrm{CI}$ & $30.3-53.0$ & $12.0-30.0$ & & $21.1-33.3$ & $9.0-18.4$ & \\
\hline $\begin{array}{l}\text { Disease control rate: } \mathrm{CR}+\mathrm{PR}+\mathrm{SD} \\
n(\%)\end{array}$ & $64(94.1)$ & $54(75.0)$ & 0.0021 & $152(76.8)$ & $113(56.5)$ & $<0.0001$ \\
\hline $95 \% \mathrm{CI}$ & $85.8-97.7$ & $63.9-83.6$ & & $70.4-82.1$ & $49.6-63.2$ & \\
\hline
\end{tabular}

$C I$ confidence interval, $C R$ complete response, $P D$ progressive disease, $P L$ placebo, $P R$ partial response, $P T X$ paclitaxel, $R A M$ ramucirumab, $S D$ stable disease

a Two-sided Cochran-Mantel-Haenszel test adjusted for randomization strata (time to progression with first-line therapy, and disease measurability

In the Japanese population, the median PFS was 5.6 months in the RAM + PTX arm versus 2.8 months in the PL + PTX arm (HR, 0.50; $95 \%$ CI, 0.35-0.73; Fig. 2c). In the Western population, the median PFS was 4.2 months in the RAM + PTX arm versus 2.8 months in the PL + PTX arm (HR, 0.63; $95 \%$ CI, 0.51-0.79; Fig. 2d).

In the Japanese population, the ORR was $41.2 \%$ (95\% CI, 30.3-53.0 \%) in the RAM + PTX arm versus $19.4 \%$ (95\% CI, 12.0-30.0\%) in the PL + PTX arm, and the DCR was $94.1 \%(95 \%$ CI, 85.8-97.7\%) in the RAM + PTX arm versus 75.0\% (95\% CI, 63.9-83.6 \%) in the PL + PTX arm (Table 3). In the Western population, the ORR was $26.8 \%(95 \% \mathrm{CI}, 21.1-33.3 \%)$ in the RAM + PTX arm versus $13.0 \%(95 \% \mathrm{CI}, 9.0-18.4 \%)$ in the PL + PTX arm, and the DCR was $76.8 \%(95 \% \mathrm{CI}$, 70.4-82.1\%) in the RAM + PTX arm versus $56.5 \%$ (95\% CI, 49.6-63.2\%) in the PL + PTX arm (Table 3).

In the Japanese population, almost all of the patients in the RAM + PTX arm had tumor size reductions, as did most of the patients in the Western population, as shown in the waterfall plot in Fig. 4.

\section{Quality of life and performance status}

In the Japanese population, the percentages of patients reporting stable or improved quality-of-life scores were similar between the treatment arms at week 6 and at the end of treatment, but were higher in the RAM + PTX arm than in the PL + PTX arm at all other postbaseline assessments. In the Western population, the percentages of patients reporting stable or improved quality-of-life scores were higher in the RAM + PTX arm than in the PL + PTX arm for all on-study assessments, but were similar at the end of treatment. In the Japanese population, the data suggest a longer time to deterioration to an ECOG PS of 2 or higher for RAM + PTX therapy, with a HR of 0.64 (95\% CI, 0.29-1.40). In the Western population, the HR for the time to deterioration to an ECOG PS of 2 or higher was 0.89 (95\% CI, 0.64-1.22).

\section{Discussion}

The safety profile of RAM + PTX was comparable between the Japanese and Western populations and consistent with the safety profile seen in the RAINBOW overall results [6]. Neutropenia was one of the most frequently reported adverse events in both treatment arms, and the incidence in the PL + PTX arm was within the range of that reported in other Japanese and Western trials with a similar paclitaxel dose and schedule [7, 11-13]. Neutropenia was commoner in the Japanese population, but this could be managed safely, as indicated by the longer median duration of therapy in the RAM + PTX arm in Japanese patients, similar median relative dose intensity of ramucirumab in the two populations, and the low rates of discontinuation due to adverse events in the RAM + PTX arm in both populations. A previous phase I study [14] (with limited sample size) showed no clear difference in the pharmacokinetics of ramucirumab between 
a

Japanese $(\mathrm{N}=35)$

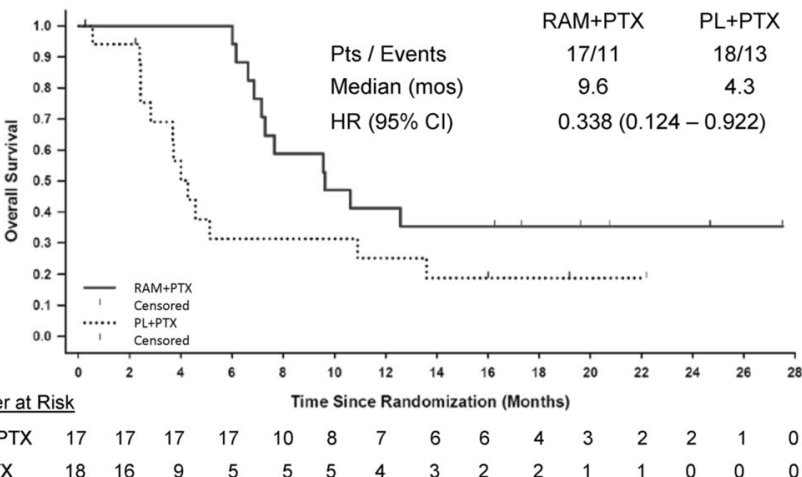

C

Japanese $(\mathrm{N}=105)$

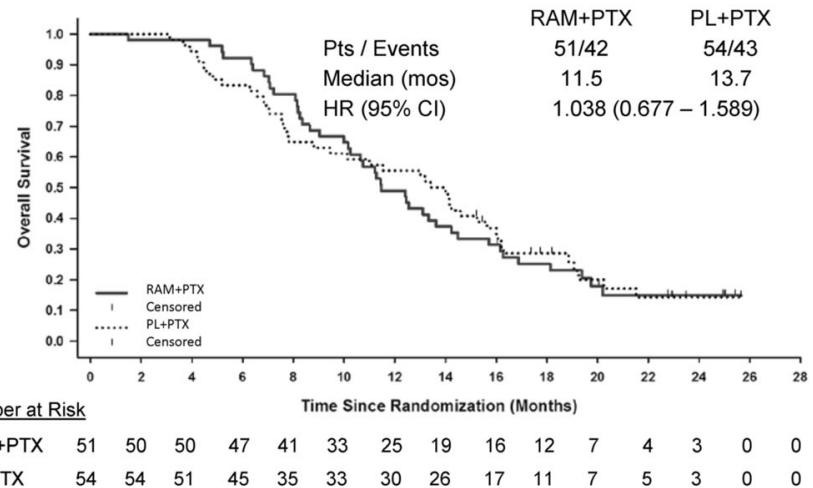

Fig. 3 Kaplan-Meier estimates of overall survival for Japanese patients (a) and Western patients (b) without post-discontinuation therapy (PDT) and of overall survival for Japanese patients (c) and

Japanese and Western patients, so the reason for bone marrow suppression is unclear. The current observations are consistent with previous studies reporting higher incidences of grade 3 or higher neutropenia associated with weekly paclitaxel therapy in Japanese patients than in Western patients $[11-13,15,16]$. Notably, the relative increase in the incidence of grade 3 or higher neutropenia when ramucirumab was added to paclitaxel therapy was similar between Japanese and Western patients, and the incidence of febrile neutropenia was low and similar between the arms in both populations. Proteinuria was commoner in the Japanese population; however, most of the events were mild and manageable. No nephrotic syndrome was reported in this study.

The pivotal phase III RAINBOW study demonstrated statistically significant benefits of RAM + PTX versus PL + PTX in OS (HR, 0.807), PFS (HR, 0.635), and ORR (28\% vs $16 \%$ ) [6]. In the present subpopulation analysis, benefits in PFS and ORR were observed in the RAM + PTX arm compared with the PL + PTX arm in b Western $(\mathrm{N}=\mathbf{2 5 0})$

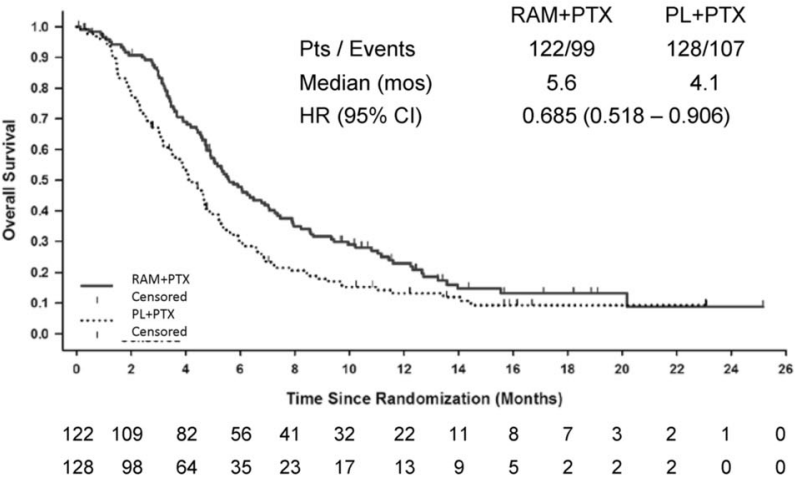

d

Western $(\mathrm{N}=148)$

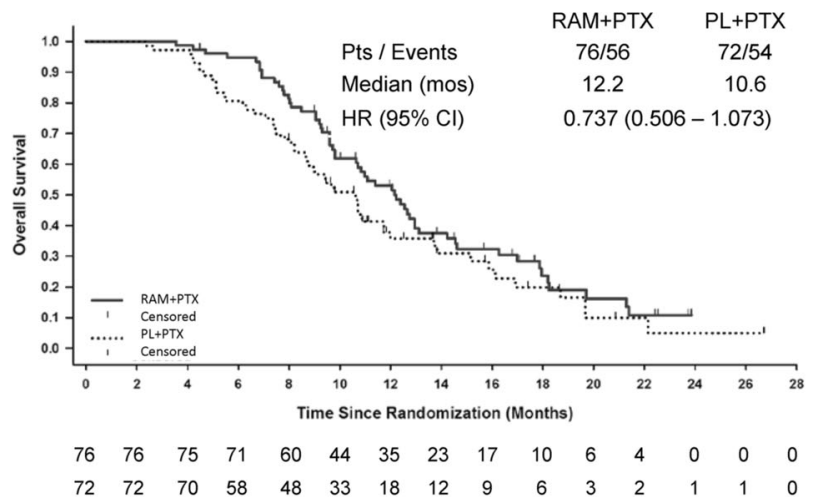

Western patients (d) with PDT. The analyses presented here are exploratory in nature. $C I$ confidence interval, $H R$ hazard ratio, mos months, $P L$ placebo, $P t s$ patients, $P T X$ paclitaxel, $R A M$ ramucirumab

Japanese and Western patients. A clearer survival benefit was observed in the Western population. The difference in OS was less clear in the Japanese subset, although the 6-month OS rate was higher in the RAM + PTX arm..

A difference in survival benefit from biological agents in Japanese/East Asian (predominantly Japanese and South Korean) patients versus Western patients with gastric cancer has been previously reported in the AVAGAST study, a randomized phase III trial evaluating bevacizumab in combination with first-line chemotherapy [17, 18]. Tumor biology (e.g., differences in plasma vascular endothelial growth factor levels and tumor neuropilin 1 expression) has been suggested as a possible explanation [17]. However, in contrast to the AVAGAST study, PFS and ORR benefits were seen in both the Japanese population and the Western population in the RAINBOW study. In addition, median survival in the PL + PTX arm was longer in the Japanese population (11.5 months) than in the Western population (5.9 months) in our study. This is in accordance with 

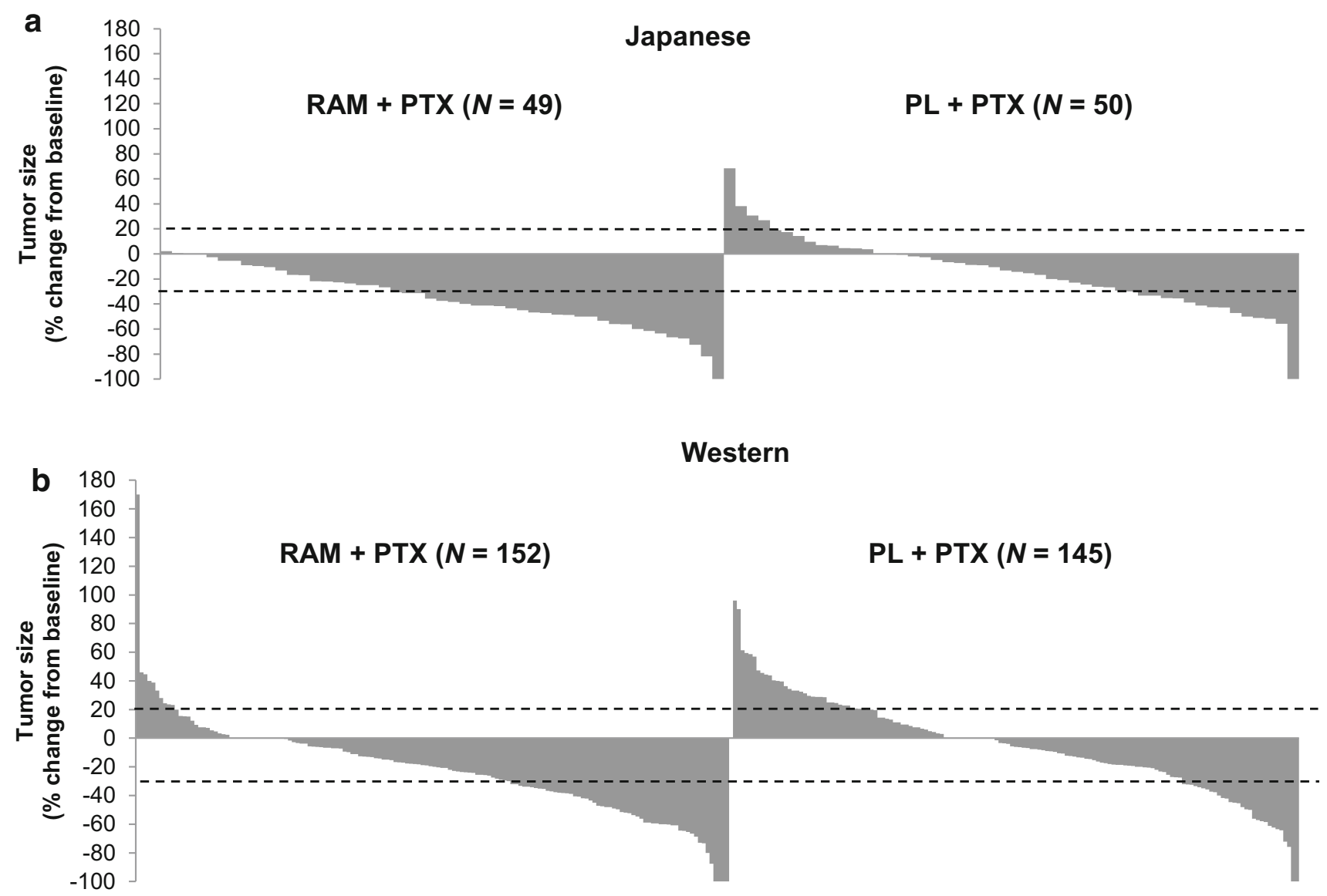

Fig. 4 Change in tumor size in Japanese patients (a) and Western patients (b). The analyses presented here are exploratory in nature. $P L$ placebo, PTX paclitaxel, RAM ramucirumab

previous studies in the first-line and second-line setting reporting better survival outcome in Asian/Japanese patients than in Western patients [3, 4, 7, 18, 19].

In the present study, there were differences between the Japanese and Western patients in some important baseline characteristics (such as ECOG PS, TTP with first-line therapy, and number of metastatic sites) indicating that the Japanese patients may have been healthier (with less tumor burden) at the start of the trial. This difference between these patient populations has been noted previously [19, 20]. Thus, patient characteristics may have contributed to the longer OS observed in the Japanese patients.

It is also possible that poststudy factors impacted the survival outcome in both populations. Whereas the use of systemic PDT was balanced between the treatment arms overall, there was a higher use of PDT in Japanese patients than in Western patients. The notably higher rate of PDT use in Japanese patients has been reported previously [19, 20]. The most commonly used chemotherapy agents in both populations are known to be active in gastric cancer, and their extensive use in the Japanese population, in particular, possibly decreased the relative survival benefit associated with ramucirumab [21]. This hypothesis is supported by exploratory survival analyses evaluating the impact of PDT use on survival. The OS difference between treatment arms was larger for patients who did not receive PDT than for patients who received PDT, and this was true for the Japanese and Western subgroups.

The quality-of-life analysis suggests that the benefits from treatment with RAM + PTX were achieved with preserved or even improved quality of life in both Japanese and Western patients. In addition, these results suggest that patients treated with RAM + PTX maintained their PS for longer in both populations.

The main limitation of these analyses is that the original clinical trial was not designed or powered to show significance in exploratory subgroups, and there was a relatively small number of patients in the Japanese population. Therefore, these results should be interpreted with caution.

In conclusion, the safety profiles of the RAM + PTX arm were similar between populations, although there was a higher incidence of neutropenia in Japanese patients. PFS and ORR improvements were observed for RAM + PTX therapy in both populations. 
Acknowledgments This work was funded by Eli Lilly and Company. We thank the patients, their families, the study sites, and the study personnel who participated in this clinical trial. We also thank Jiangang Jameson Cai of Eli Lilly, who provided the statistical programming support for this work, and Kenjiro Fukase, who assisted with data analysis and interpretation. Eli Lilly and Company contracted inVentiv Health Clinical for writing and editorial support, provided by Jody Arsenault and Noelle Gasco, respectively.

\section{Compliance with ethical standards}

Conflict of interest Yukako Ichimiya works for Eli Lilly Japan. Michael Emig works for Eli Lilly and Company. Astra M. Liepa works for and owns stock in Eli Lilly and Company. Kumari Chandrawansa works for Eli Lilly and Company. An immediate family member of Atsushi Ohtsu is an employee of Celgene. Shuichi Hironaka has received honoraria from Taiho Pharmaceutical, Novartis Pharmaceuticals, Takeda Pharmaceutical, and Yakult Honsha, has had a consulting or advisory role for Yakult Honsha and Eli Lilly and Company, and has received travel and accommodation expenses from AstraZeneca and Eli Lilly and Company. Yoshihiko Segawa has received honoraria from Taiho Pharmaceutical, Boehringer Ingelheim, Chugai Pharmaceutical, Nippon Kayaku, BristolMyers Squibb, and Kyowa Kirin. Yasuhiro Shimada has received honoraria from Eli Lilly and Company, Taiho Pharmaceutical, Chugai Pharmaceutical, Yakult Honsha, Pfizer, Bayer, Bristol-Myers Squibb, and Merck Serono and research funding from Eli Lilly and Company, Taiho Pharmaceutical, Chugai Pharmaceutical, Yakult Honsha, Pfizer, Sanofi, and Novartis Pharmaceuticals. Fumio Nagashima has received honoraria and research funding from Eli Lilly Japan. Kei Muro has served on speakers' bureaus for Eli Lilly and Company and Bristol-Myers Squibb. Kensei Yamaguchi has served on speakers' bureaus for Merck Serono and Chugai Pharmaceutical. Tomohiro Nishina has served on speakers' bureaus for Taiho Pharmaceutical, Takeda Pharmaceutical, Merck Serono, Bayer, and Chugai Pharmaceutical. Yasushi Omuro, Taito Esaki, Naotoshi Sugimoto, Hirofumi Yasui, Seigo Yukisawa, Kohei Shitara, Yoshito Komatsu, Takao Tamura, Masahiro Gotoh, Toshihiko Doi, and Hansjochen Wilke declare that they have no conflict of interest.

\section{References}

1. Ferlay J, Soerjomataram I, Ervik M, et al. GLOBOCAN 2012 v1.0. Cancer incidence and mortality worldwide: IARC cancer base no. 11 (Internet). Lyon: International Agency for Research on Cancer. http://globocan.iarc.fr/Pages/fact_sheets_cancer.aspx.

2. Wagner AD, Grothe W, Haerting J, Kleber G, Grothey A, Fleig WE. Chemotherapy in advanced gastric cancer: a systematic review and meta-analysis based on aggregate data. J Clin Oncol. 2006;24:2903-9.

3. Thuss-Patience PC, Kretzschmar A, Bichev D, Deist T, Hinke A, Breithaupt K, et al. Survival advantage for irinotecan versus best supportive care as second-line chemotherapy in gastric cancer-a randomised phase III study of the Arbeitsgemeinschaft Internistische Onkologie (AIO). Eur J Cancer. 2011;47:2306-14.

4. Ford HE, Marshall A, Bridgewater JA, Janowitz T, Coxon FY, Wadsley J, COUGAR-02 Investigators, et al. Docetaxel versus active symptom control for refractory oesophagogastric adenocarcinoma (COUGAR-02): an open-label, phase 3 randomised controlled trial. Lancet Oncol. 2014;15:78-86.

5. Kang JH, Lee SI, Lim DH, Park KW, Oh SY, Kwon HC, et al. Salvage chemotherapy for pretreated gastric cancer: a randomized phase III trial comparing chemotherapy plus best supportive care with best supportive care alone. J Clin Oncol. 2012;30:1513-8.

6. Wilke H, Muro K, Van Cutsem E, Oh SC, Bodoky G, Shimada Y, et al. Ramucirumab plus paclitaxel versus placebo plus paclitaxel in patients with previously treated advanced gastric or gastrooesophageal junction adenocarcinoma (RAINBOW): a doubleblind, randomised phase 3 trial. Lancet Oncol. 2014;15:1224-35.

7. Hironaka S, Ueda S, Yasui $H$, Nishina T, Tsuda M, Tsumura T, et al. Randomized, open-label, phase III study comparing irinotecan with paclitaxel in patients with advanced gastric cancer without severe peritoneal metastasis after failure of prior combination chemotherapy using fluoropyrimidine plus platinum: WJOG 4007 trial. J Clin Oncol. 2013;31:4438-44.

8. Ohtsu A, Yoshida S, Saijo N. Disparities in gastric cancer chemotherapy between the East and West. J Clin Oncol. 2006;24:2188-96.

9. Kim R, Tan A, Choi M, El-Rayes BF. Geographic differences in approach to advanced gastric cancer: is there a standard approach? Crit Rev Oncol Hematol. 2013;88:416-26.

10. Aaronson NK, Ahmedzai S, Bergman B, Bullinger M, Cull A, Duez NJ, et al. The European Organization for Research and Treatment of Cancer QLQ-C30: a quality-of-life instrument for use in international clinical trials in oncology. J Natl Cancer Inst. 1993;85:365-76.

11. Hironaka S, Zenda S, Boku N, Fukutomi A, Yoshino T, Onozawa Y. Weekly paclitaxel as second-line chemotherapy for advanced or recurrent gastric cancer. Gastric Cancer. 2006;9:14-8.

12. Kodera $\mathrm{Y}$, Ito $\mathrm{S}$, Mochizuki $\mathrm{Y}$, Fujitake $\mathrm{S}$, Koshikawa $\mathrm{K}$, Kanyama Y, et al. A phase II study of weekly paclitaxel as second-line chemotherapy for advanced gastric cancer (CCOG0302 study). Anticancer Res. 2007;27(4C):2667-71.

13. Shitara K, Matsuo K, Takahari D, Yokota T, Shibata T, Ura T, et al. Neutropenia as a prognostic factor in advanced gastric cancer patients undergoing second-line chemotherapy with weekly paclitaxel. Ann Oncol. 2010;21:2403-9.

14. Ueda S, Satoh T, Gotoh M, Gao L, Doi T. A phase Ib study of safety and pharmacokinetics of ramucirumab in combination with paclitaxel in patients with advanced gastric adenocarcinomas. Oncologist. 2015;20:493-4.

15. Del Mastro L, Perrone F, Repetto L, Manzione L, Zagonel V, Fratino L, et al. Weekly paclitaxel as first-line chemotherapy in elderly advanced breast cancer patients: a phase II study of the Gruppo Italiano di Oncologia Geriatrica (GIOGer). Ann Oncol. 2005;16:253-8.

16. Seidman AD, Berry D, Cirrincione C, Harris L, Muss H, Marcom PK, et al. Randomized phase III trial of weekly compared with every-3-weeks paclitaxel for metastatic breast cancer, with trastuzumab for all HER-2 overexpressors and random assignment to trastuzumab or not in HER-2 nonoverexpressors: final results of Cancer and Leukemia Group B protocol 9840. J Clin Oncol. 2008;26:1642-9.

17. Van Cutsem E, de Haas S, Kang YK, Ohtsu A, Tebbutt NC, Ming $\mathrm{XuJ}$, et al. Bevacizumab in combination with chemotherapy as first-line therapy in advanced gastric cancer: a biomarker evaluation from the AVAGAST randomized phase III trial. J Clin Oncol. 2012;30:2119-27.

18. Ohtsu A, Shah MA, Van Cutsem E, Rha SY, Sawaki A, Park SR, et al. Bevacizumab in combination with chemotherapy as firstline therapy in advanced gastric cancer: a randomized, doubleblind, placebo-controlled phase III study. J Clin Oncol. 2011;29:3968-76

19. Sawaki A, Ohashi Y, Omuro Y, Satoh T, Hamamoto Y, Boku N, et al. Efficacy of trastuzumab in Japanese patients with HER2positive advanced gastric or gastroesophageal junction cancer: a subgroup analysis of the Trastuzumab for Gastric Cancer (ToGA) study. Gastric Cancer. 2012;15:313-22. 
20. Nishina T, Sawaki A, Yamada Y, Yamaguchi K, Doi T, Chin K, et al. Regional differences in patient characteristics of AVAGAST: An exploratory comparison between Japanese patients and rest of world patients in the placebo arm. Poster presented at the American Society of Clinical Oncology, Chicago, 1-5 June, 2012.
21. Korn EL, Freidlin B, Abrams JS. Overall survival as the outcome for randomized clinical trials with effective subsequent therapies. J Clin Oncol. 2011;29:2439-42. 